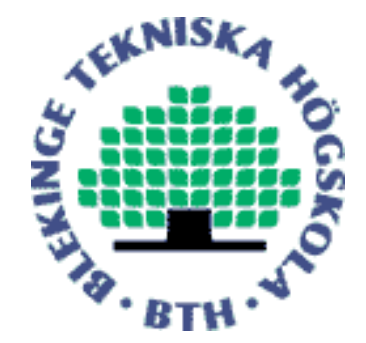

Copyright (C) 2013 IEEE.

Citation for the published paper:

Moving Target Focusing with Normalized Relative Speed in Azimuth-Invarian Bistatic Sar

Viet Thuy Vu, Thomas Sjögren, Mats Pettersson

International Geoscience and Remote Sensing Symposium

2013 Melbourne

This material is posted here with permission of the IEEE. Such permission of the IEEE does not in any way imply IEEE endorsement of any of BTH's products or services Internal or personal use of this material is permitted. However, permission to reprint/republish this material for advertising or promotional purposes or for creating new collective works for resale or redistribution must be obtained from the IEEE by sending a blank email message to pubs-permissions@iee.org.

By choosing to view this document, you agree to all provisions of the copyright laws protecting it. 


\title{
MOVING TARGET FOCUSING WITH NORMALIZED RELATIVE SPEED IN AZIMUTH-INVARIAN BISTATIC SAR
}

\author{
Viet T. Vu, Thomas K. Sjögren, Mats I. Pettersson \\ Blekinge Institute of Technology, Campus Gräsvik, 37179 Karlskrona, Sweden
}

\begin{abstract}
Focusing moving targets with Normalized Relative Speed (NRS)for bistatic synthetic aperture radar (SAR) is discussed in this paper. The discussion concentrates on azimuthinvariant bistatic geometry. The focusing approach for azimuth-invariant bistatic geometry is derived analytically. The validity of the proposed approach for other bistatic geometry like azimuth-variant is also investigated.
\end{abstract}

Index Terms - SAR, bistatic, moving target, focusing, NRS

\section{INTRODUCTION}

Bistatic SAR refers to SAR systems whose transmitter and receiver(s) are separated. This allows bistatic SAR having some advantages over monostatic SAR like reducing vulnerability for military applications. Based on the relative position of transmitter with respect to receiver, bistatic geometries are categorized: quasi-monostatic, azimuth-invariant and general or azimuth-variant. For quasi-monostatic geometry, transmitter and receiver are mounted on a single platform whereas transmitter and receiver are separated in two different platforms for azimuth-invariant and azimuth-variant geometries. The research presented in this paper concentrates on the azimuth-invariant geometry that the relative position of transmitter with respect to receiver is a constant.

Unique advantages of bistatic SAR motivate a large number of researches on bistatic SAR. The main research topic of bistatic SAR is bistatic algorithms. Hence, different monostatic time- and frequency-domain algorithms have been applied directly or extended to bistatic cases which allow building SAR scenes illuminated by bistatic SAR systems. Research on applications for bistatic SAR like Ground Moving Target Indication (GMTI) is emerged recently.

A combination of space time adaptive processing (STAP) and bistatic SAR is presented in [1]. This combination is seen as a promising technique for bistatic GMTI. However, focusing a moving target in a SAR image is not included in this bistatic GMTI technique. Similar to monostatic SAR, a moving target in bistatic SAR is be smeared and appear in the SAR image as a curve due to the complex range migration. For relatively short synthetic apertures, the smear can be ne- glected since a moving target is seen to be stationary due to the small movement during the integration time. In this case, the moving target is approximately focused in the SAR image as shown in [1]. For long synthetic apertures, the movement of a target can be significant and the smearing effect cannot be avoided. A focusing approach is therefore desired to refocus the moving target in the SAR image.

The aim of this research is to derive a focusing approach for bistatic SAR systems analytically. Although azimuthinvariant bistatic geometry is concentrated other bistatic geometries are also taken into account.

The paper is organized as follows. Section II derives the moving target focusing approach for bistatic SAR with emphasis on azimuth-invariant geometry. The equations derived in Section II are verified by the simulations in Section III. Section IV presents the extensions of the focusing approach to other bistatic geometries. Section V provides the conclusions.

\section{MOVING TARGET FOCUSING APPROACH FOR AZIMUTH-INVARIANT BISTATIC SAR}

Let's consider a bistatic SAR system attached with a Cartesian coordinate system $(\xi, \eta, \zeta)$ as illustrated in Fig. 1. The movements of the platforms is assumed to be linear with constant speeds. For the transmitting platform, this movement can be expressed by the following set of equations

$$
\begin{aligned}
& \xi_{T}(t)=v_{T, \xi} t+\xi_{T, 0} \\
& \eta_{T}(t)=v_{T, \eta} t+\eta_{T, 0} \\
& \zeta_{T}(t)=\zeta_{T, 0}
\end{aligned}
$$

where $v_{T, \xi}$ and $v_{T, \eta}$ are the transmitting platform speed components. The initial coordinates of the transmitting platform in a Cartesian coordinate system are given by $\left(\xi_{0}, \eta_{0}, \zeta_{0}\right)$ in which $\zeta_{0}$ is the flight altitude of the platform. Similarly, the expression of the movement of the receiving platform is given by

$$
\begin{aligned}
& \xi_{R}(t)=v_{R, \xi} t+\xi_{R, 0} \\
& \eta_{R}(t)=v_{R, \eta} t+\eta_{R, 0} \\
& \zeta_{R}(t)=\zeta_{R, 0}
\end{aligned}
$$

where the variables have the same meanings as those of (1). The difference is only that the subscript $T$, i.e. transmitting, 


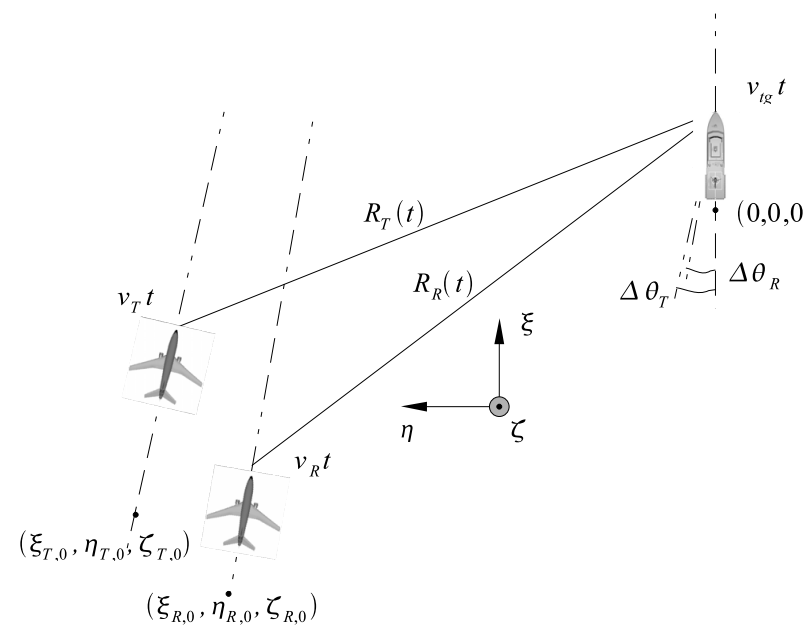

Fig. 1. Bistatic SAR system geometry.

is replaced by the subscript $R$, i.e. receiving. This bistatic SAR system is assumed to illuminate a ground scene where there exists a moving target. The movement of the target on the ground is also assumed to be linear with constant speeds. Without losing generality, this movement of the target can simply be expressed by

$$
\begin{aligned}
& \xi_{t g}(t)=v_{t g} t \\
& \eta_{t g}(t)=0 \\
& \zeta_{t g}(t)=0
\end{aligned}
$$

where $v_{t g}$ denotes the speed of the target in the ground scene. The true bistatic range - the traveling distance of a radar pulse originated from the transmitter impinging on a target on the SAR scene and scattered to the receiver - is calculated as well as analyzed by

$$
\begin{aligned}
R_{\text {true }}(t) & =\sqrt{\left[\xi_{T}(t)-\xi_{t g}(t)\right]^{2}+\eta_{T}^{2}(t)+\zeta_{T, 0}^{2}} \\
& +\sqrt{\left[\xi_{R}(t)-\xi_{t g}(t)\right]^{2}+\eta_{R}^{2}(t)+\zeta_{R, 0}^{2}}
\end{aligned}
$$

If the true bistatic range is used to reconstruct a SAR image, the moving target will be smeared as a result of the complex range migration. According to the concept of the relative speed between two objects in motion, one moving object is seen to be stationary for the other which moves with the relative speed between them. Thus, a moving target can be seen to be stationary for the SAR platform if the platform is assumed to move with the relative speed. This principle has been used to focus moving targets in SAR image for monostatic SAR. This principle is supposed to be also valid for bistatic SAR. Hence, in the SAR image formation process, if the considered moving target is displaced and refocused at the coordinates $\left(x_{0}, y_{0}\right)$ in a image coordinate system $(x, y, z)$, there will be a pair NRS, i.e. $\left(\gamma_{T}, \gamma_{R}\right)$, which satisfies the following cir- cumstance during the integration time

$$
\begin{aligned}
R_{\text {est }} & (t)=R_{\text {true }}(t) \\
& =\sqrt{\left[\gamma_{T} \xi_{T}(t)-x_{0}\right]^{2}+\left[\gamma_{T} \eta_{T}(t)-y_{0}\right]^{2}+\zeta_{T, 0}^{2}} \\
& +\sqrt{\left[\gamma_{R} \xi_{R}(t)-x_{0}\right]^{2}+\left[\gamma_{R} \eta_{R}(t)-y_{0}\right]^{2}+\zeta_{R, 0}^{2}}
\end{aligned}
$$

In other word, $\left(\gamma_{T}, \gamma_{R}\right)$ is supposed to result in an estimated bistatic range which is identical the true range history given in (4). Equation (5) is so-called the moving target focusing equation for bistatic SAR. Substituting (4) into (5) results in an equation with the mathematical form of $\sqrt{a}+\sqrt{b}=\sqrt{c}+$ $\sqrt{d}$. To solve such equation, we can take the square both sides of the equation until the square roots disappear. The final presentation of this equation can easily be show to be

$$
\begin{aligned}
& (a+b-c-d)^{4}+16(a b-c d)^{2} \\
& \quad-8(a+b-c-d)^{2}(a b+c d)=0
\end{aligned}
$$

and then

$$
\begin{aligned}
A t^{8}+B t^{7} & +C t^{6}+D t^{5}+E t^{4} \\
& +F t^{3}+G t^{2}+H t+I=0
\end{aligned}
$$

Equation (7) is an eighth order equation. To be assure that the condition (7) satisfies at any time $t$ during the integration time, the following condition must be fulfilled

$$
A=B=C=D=E=F=G=H=I=0
$$

The equation $A=0$ corresponding to the leading term $t^{8}$ is shown to be

$$
\begin{aligned}
& \gamma_{T} v_{T}-\sqrt{\left(v_{T, \xi}-v_{t g}\right)^{2}+\left(v_{T, \eta}\right)^{2}} \\
& \quad+\gamma_{R} v_{R}-\sqrt{\left(v_{R, \xi}-v_{t g}\right)^{2}+\left(v_{R, \eta}\right)^{2}}=0
\end{aligned}
$$

Equation (9) is a first order and two variable equation and there exist different pairs of $\left(\gamma_{T}, \gamma_{R}\right)$ which can meet the demand of (9). Among possible roots of (9), a deductive root based on the available knowledge of the monostatic case is

$$
\gamma_{T}=\frac{\sqrt{\left(v_{T, \xi}-v_{t g}\right)^{2}+\left(v_{T, \eta}\right)^{2}}}{v_{T}}
$$

and

$$
\gamma_{R}=\frac{\sqrt{\left(v_{R, \xi}-v_{t g}\right)^{2}+\left(v_{R, \eta}\right)^{2}}}{v_{R}}
$$

For this pair of $\left(\gamma_{T}, \gamma_{R}\right)$, the equation $B=0$ corresponding to the next leading term, i.e. $t^{7}$ is also fulfilled. Other equations corresponding to the terms $t^{0} \cdots t^{6}$ are satisfied if and only if

$$
\left\{\begin{array}{l}
v_{T, \xi}=v_{R, \xi}=v_{p l, \xi} \\
v_{T, \eta}=v_{R, \eta}=v_{p l, \eta} \\
\xi_{T, 0}=\xi_{R, 0}=\xi_{0} \\
\eta_{T, 0}=\eta_{R, 0}=\eta_{0}
\end{array}\right.
$$


Table 1. Parameters of the simulated platforms.

\begin{tabular}{ccc}
\hline \hline Parameter & Transmitter & Receiver \\
\hline Maximum frequency & $22 \mathrm{MHz}$ & \\
Minimum frequency & $82 \mathrm{MHz}$ & \\
Platform speed & $126 \mathrm{~m} / \mathrm{s}$ & $=$ \\
Flight altitude & $4944 \mathrm{~m}$ & $2894 \mathrm{~m}$ \\
Minimum range & $5891 \mathrm{~m}$ & $4317 \mathrm{~m}$ \\
PRF & $137 \mathrm{~Hz}$ & \\
Aperture step & $0.9375 \mathrm{~m}$ & \\
\hline No. aperture positions & 8192 & $=$ \\
Direction of movement & $25^{\circ}$ & $=$ \\
Initial coordinates & $(-1578,-3204)$ & $=$ \\
\hline \hline
\end{tabular}

Table 2. Parameters of simulated targets in ground scene.

\begin{tabular}{ccc}
\hline \hline Parameter & Target 0 & Target 1 \\
\hline Scatterer's feature & Point-like & Point-like \\
Target speed & $0 \mathrm{~m} / \mathrm{s}$ & $5 \mathrm{~m} / \mathrm{s}$ \\
Initial coordinates & $(64,-64)$ & $(0,0)$ \\
Direction of movement & $0^{\circ}$ \\
\hline NRS & 0.9642 \\
Refocusing coordinates & $\approx(-66,55)$ \\
\hline \hline
\end{tabular}

The set of equation (12) is fulfilled when the velocities of the the platforms and their initial coordinates are identical or in other words the bistatic geometry is azimuth-invariant. In this case, The coordinates $\left(x_{0}, y_{0}\right)$ where a target is refocused in a SAR image are given by

$$
\begin{aligned}
& x_{0}=\frac{1}{\gamma}\left[\xi_{0}(\gamma-1)+\frac{v_{t g}\left(v_{p l, \xi} \xi_{0}-v_{p l, \eta} \eta_{0}\right)}{v_{p l}^{2}}\right] \\
& y_{0}=\frac{1}{\gamma}\left[\eta_{0}(\gamma-1)+\frac{v_{t g}\left(v_{p l, \xi} \eta_{0}+v_{p l, \eta} \xi_{0}\right)}{v_{p l}^{2}}\right]
\end{aligned}
$$

\section{VERIFICATION}

In this section, we present some simulation results to verify (10), (11), (13) and (14). The simulated bistatic SAR system is based on CARABAS-II's parameters in reality [2]. The system parameters are summarized in the upper part of Table I. Other parameters used in the simulation can be found in the lower part of Table I. The ground scene includes one pointlike scatterer located constantly in about the middle of the

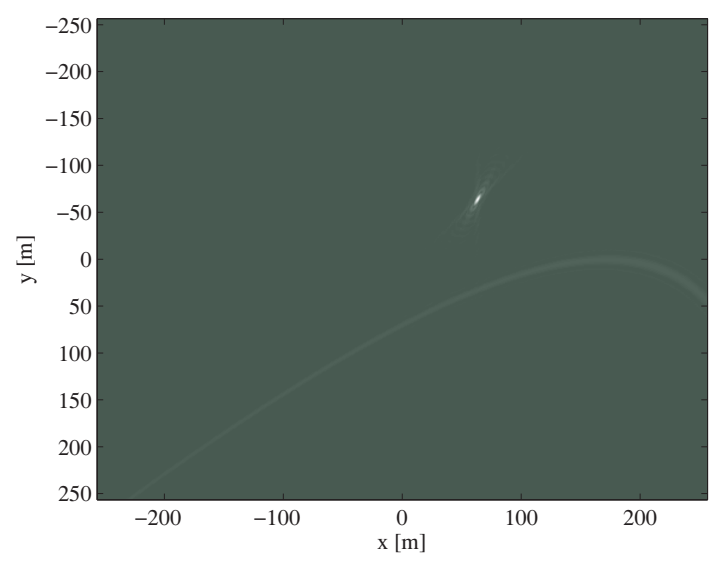

Fig. 2. SAR image of the simulated ground scene.

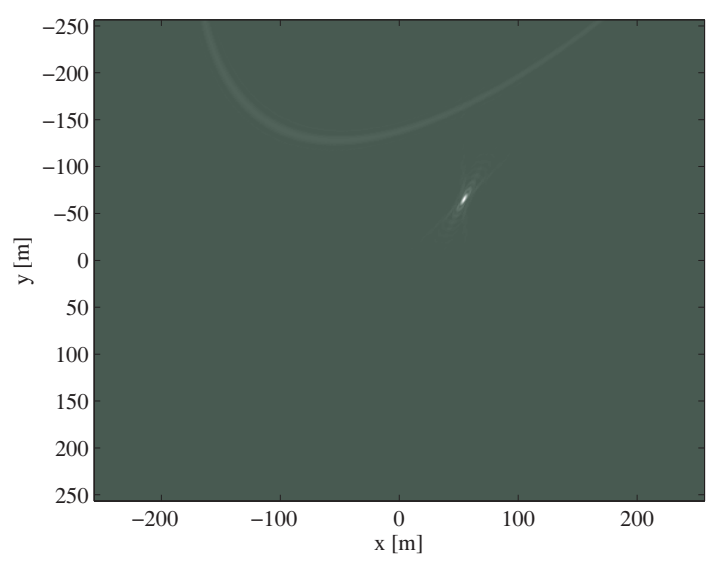

Fig. 3. SAR image with correctly focused moving target.

scene (so-called the stationary target) and one random moving point-like scatterer (so-called the moving target). The parameters of the simulated target in the ground scene are given in the upper part of Table 2. The lower part of Table 2 is reserved for the latter calculation results. Fig. 2 shows the SAR image of the simulated ground scene with the Bistatic Global Backprojection (BiGBP) algorithm [3] using the true bistatic range (4). As shown, the stationary target is well focused and appears in about the middle of the SAR image as a point target while the moving target is smeared and appears as a curve in the lower part of the SAR image. The coordinates of the stationary target in the SAR image is matched to its true coordinates in the simulation.

To examine (10) and (11), we use the focusing equation (5) where $\gamma_{T}$ and $\gamma_{R}$ are given by (10) and (11), respectively. From the parameters given in Table 1 and the higher part of Table 2, (10) and (11) result in the values $\gamma_{T}=\gamma_{R}=0.9642$. 


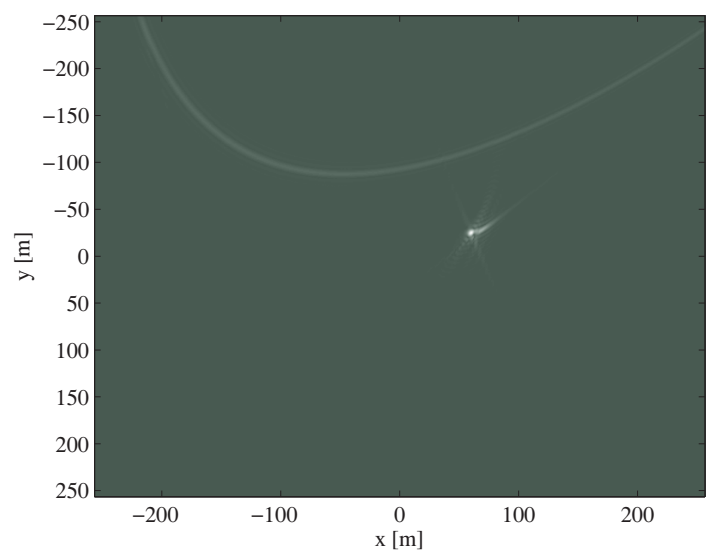

Fig. 4. SAR image with approximately focused moving target in the case of azimuth-invariant without initial assumption.

The new SAR image with moving target focusing is given in Fig. 3. We can see that the moving target is refocused as a point target in about the middle of the SAR image whereas the stationary target gets blurred as curve and displaced to the upper part of the SAR image. This results show the exact of (10) and (11).

To verify (13) and (14), we first estimate the coordinates, where the moving targets are refocused, given by them and then compare with the coordinates in Fig. 3. With the simulation parameters, (13) and (14) result in the coordinates $(-65.7437,55.1527)$ where the target should be refocused. This result is almost identical to the true coordinates of the refocused moving target in the SAR image in Fig. 3. The differences between the results are only due to the selected image sample.

\section{FURTHER INVESTIGATIONS}

In this section, further investigations about the moving target focusing for bistatic SAR are presented. The system parameters are modified to examine the possibilities to extend the focusing approach to different bistatic geometries. In the first extension, we consider a bistatic SAR system with azimuthinvariant geometry but without the assumption of initial coordinates. It is assumed that the transmitter platform stays far from the SAR scene to reduce vulnerability. Hence, the initial coordinates of the transmitter platform is changed to $(-1578,-5204)$, i.e. $2000 \mathrm{~m}$ separation in the $\eta$ direction. In this case, $\gamma_{T}$ and $\gamma_{R}$ are unchanged. Fig. 4 shows the SAR image with the approximately focused moving target. The smearing effect is not very strong but can be observed in the SAR image.

In the second extension, a bistatic SAR system with azimuth-variant geometry is considered. Hence, the the di-

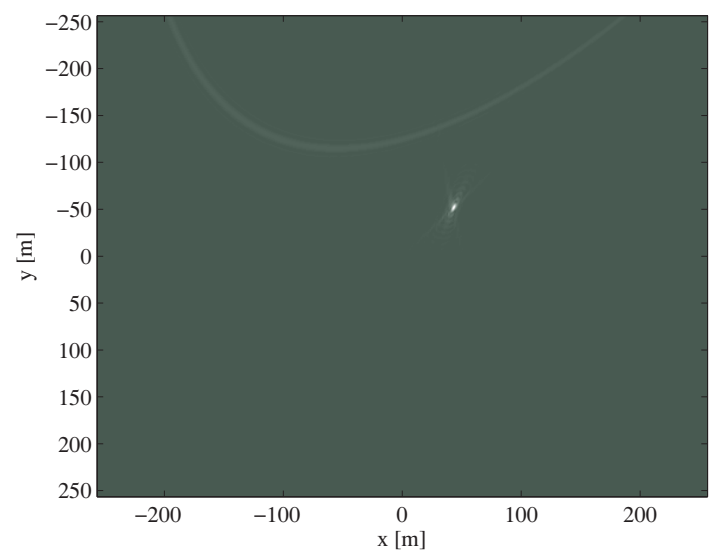

Fig. 5. SAR image with approximately focused moving target in the case of azimuth-variant.

rection of movement of the transmitter is changed to $15^{\circ}$ whereas the speed of the receiver platform is slightly increased to $130 \mathrm{~m} / \mathrm{s}$. Therefore, the new values of NRS are $\gamma_{T}=0.9617$ and $\gamma_{R}=0.9654$. The SAR image with the approximately focused moving target is given in Fig. 5. The smear happens but it is hard to observe the effect in the SAR image or the smearing effect can be neglected in this case.

\section{CONCLUSION}

In this paper, an analytical derivation of the moving target focusing approach using NRS for azimuth-invariant bistatic is presented. The approach has been verified with the simulation results. The proposal is also extended for other bistatic geometries. For the azimuth-invariant geometry, moving targets can definitely be focused in SAR image. It also is possible to approximately focus moving targets for other geometries.

\section{REFERENCES}

[1] V. T. Vu, T. K. Sjögren, and M. I. Pettersson, "Space time adaptive processing for moving target detection and imaging in bistatic SAR ," in Proc. IEEE IGARSS'2011, Vancouver, Canada, Jul. 2011, pp. 28292832 .

[2] A. Gustavsson, L. M. H. Ulander, B. H. Flood, P.O. Frölind, H. Hellsten, T. Jonsson, B. Larsson, and G. Stenstrom, "Development and operation of an airborne VHF SAR system-lessons learned," in Proc. IEEE IGARSS'98, vol. 1, Seattle, WA, Jul. 1998, pp. 458-462.

[3] V. T. Vu, T. K. Sjögren, and M. I. Pettersson, "Fast backprojection for UWB bistatic SAR," in Proc. IEEE Radar'2011, Kansas City, MO, May 2011, pp. 431-434. 